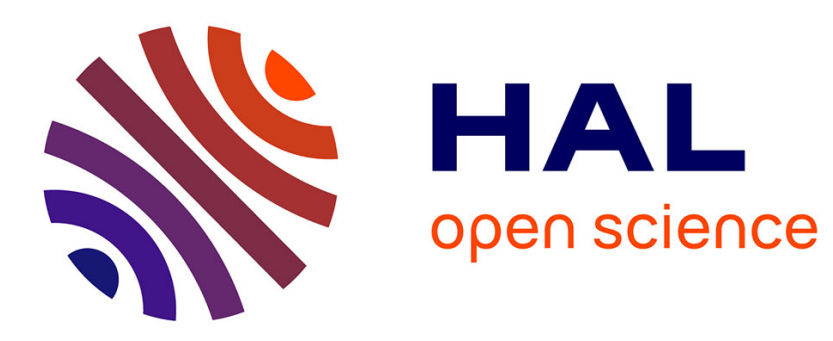

\title{
Low power all-optical bistability in InGaAs-AlInAs superlattices: demonstration of a wireless self-electro-optical effect device
}

J. Couturier, J.C Harmand, P. Voisin

\section{- To cite this version:}

J. Couturier, J.C Harmand, P. Voisin. Low power all-optical bistability in InGaAs-AlInAs superlattices: demonstration of a wireless self-electro-optical effect device. Journal de Physique IV Proceedings, 1993, 03 (C5), pp.C5-253-C5-256. 10.1051/jp4:1993549 . jpa-00251635

HAL Id: jpa-00251635

https://hal.science/jpa-00251635

Submitted on 1 Jan 1993

HAL is a multi-disciplinary open access archive for the deposit and dissemination of scientific research documents, whether they are published or not. The documents may come from teaching and research institutions in France or abroad, or from public or private research centers.
L'archive ouverte pluridisciplinaire HAL, est destinée au dépôt et à la diffusion de documents scientifiques de niveau recherche, publiés ou non, émanant des établissements d'enseignement et de recherche français ou étrangers, des laboratoires publics ou privés. 


\title{
Low power all-optical bistability in InGaAs-AlInAs superlattices: demonstration of a wireless self-electro-optical effect device
}

\author{
J. COUTURIER, J.C. HARMAND* and P. VOISIN
}

\author{
Laboratoire de Physique de la Matière Condensée de l'Ecole Normale Supérieure, 24 rue Lhomond, \\ 75005 Paris, France \\ * Centre National d'Etude des Télécommunications, Laboratoire de Bagneux, 196 av. H. Ravera, \\ 92220 Bagneux, France
}

\begin{abstract}
We report the observation of optical transmission bistability at low temperature in unprocessed InGaAs-AlInAs superlattice PIN structures. Bistability results, in analogy with the self electro-optical effect device, from a positive feedback mecanism due to the interplay between Wannier-Stark effect, built-in field and screening by photocarriers.
\end{abstract}

There is a tremendous potential interest in optical bistable devices because they are ideally suited to highly parallel information processing, commutation matrices for telecommunications, etc.. However, alloptical bistables ${ }^{1}$ based on non-linear properties of the optical index in Fabry-Pérot cavities require a relatively high power ( $>1 \mu \mathrm{W} / \mu \mathrm{m}^{2}$, i.e. more than $1 \mathrm{~mW} /$ pixel $^{2}$ ) which seriously limits cw operation and integration. On the other hand, electro-optical bistables like the self electro-optical effect device 3 (SEED) do function at low power (a few $\mu \mathrm{W}$ / device), but they need an individual electrical circuit, which implies a complex architecture of interconnections and again limits the possibility of large scale integration. Recently, the invention of the S-SEED ${ }^{4}$ has open new perspectives since this combination of two SEEDs functions with no other external electrical connection than a simple bias voltage. However, these devices still require rather complex processing ${ }^{5}$ and differential illumination which hamper their use in large scale matrices. Here, we show that all-optical bistability may be obtained at very low power and without any processing in properly designed superlattice PIN diodes, using the combination of Wannier-Stark effect, built-in electric field and screening.

The sample used in this study is a InGaAs-AlInAs superlattice electro-optical modulator structure grown by Molecular Beam Epitaxy on $n^{+}$InP substrates. It consists in a 20 period InGaAs $(60 \AA)$-AlInAs $(20 \AA)$ superlattice embedded between "confinement" layers formed by 10 -period InGaAs ( $25 \AA$ )-AlInAs $(20 \AA)$. This undoped structure, originally designed for guided-wave modulation at $1.55 \mu \mathrm{m}^{6}$, is inserted between thick AlInAs $\mathrm{n}^{+}$and $\mathrm{p}^{+}$electrodes and forms a PIN diode. In addition, the sample is topped by a $1000 \AA$ thick $\mathrm{p}^{+}$InGaAs contact layer. Low temperature $(2 \mathrm{~K})$ photocurrent spectra at $0 \mathrm{~V}$ and $0.5 \mathrm{~V}$ forward bias are shown in Fig. 1a. These spectra illustrate the now well-known scenario of the WannierStark effect 7,9 : the absorption spectrum of a biased superlattice consists in a series of transitions between discrete quantum states partially localized in the vincinity of the different quantum wells. These transitions occur at the energies $E_{0}+p$ eFd, where $E_{0}$ is essentially the bandgap of an isolated quantum well, $F$ is the electric field, $d$ the superperiod and $p$ a relative integer. The $p=0$ "vertical" transition is field-independent and corresponds to electron and hole wavefunctions centered in the same quantum well, while the fielddependent $p \neq 0$ "oblique" transitions occur between wavefunctions localized near quantum wells separated by p periods. Here, the built-in electric field $(\approx 50 \mathrm{kV} / \mathrm{cm})$ already corresponds to the "large field" regime where only the dominant vertical transition at $935 \mathrm{meV}$ and weak $\mathrm{p}=-1$ and $p=+1$ oblique transitions at 900 and $970 \mathrm{meV}$ can be observed. When the field is decreased by applying a $0.5 \mathrm{~V}$ forward bias, the $\mathrm{p}=-1$ transition is blue-shifted while its oscillator strength increases, and a weak $p=-2$ transition becomes 
observable. Near flatband conditions, all these transitions merge and form the superlattice miniband absorption spectrum. It is clear that near $920 \mathrm{meV}$, there is a region of well contrasted "blueshift" modulation where the absorption decreases when the field strength increases, while near 900 meV there is the region of low drive voltage "redshift" modulation by the oblique transitions used in the guided-wave modulators ${ }^{6}$.

Furthermore, the built-in electric field can be screened by photo-carriers. This is illustrated in Fig. Ib which shows the transmision spectrum at very low power, modulated using an additional illumination by a $0.5 \mathrm{~mW}$ He-Ne laser chopped at $75 \mathrm{~Hz}$ : this differential transmission spectrum essentially reveals the difference between the large and low field absorption spectra of Fig. 1a. In particular, the "blueshift" modulation region appears as a strong negative peak at $930 \mathrm{meV}$. This peak corresponds to a $15 \%$ change in the transmitted intensity, which is quite remarkable since the absorption on the two-dimensional plateau above $980 \mathrm{meV}$ is only $20 \%$. Hence, half a mW distributed over the $3 \times 4 \mathrm{~mm}^{2}$ sample area is sufficient to modulate $75 \%$ of the available absorption.

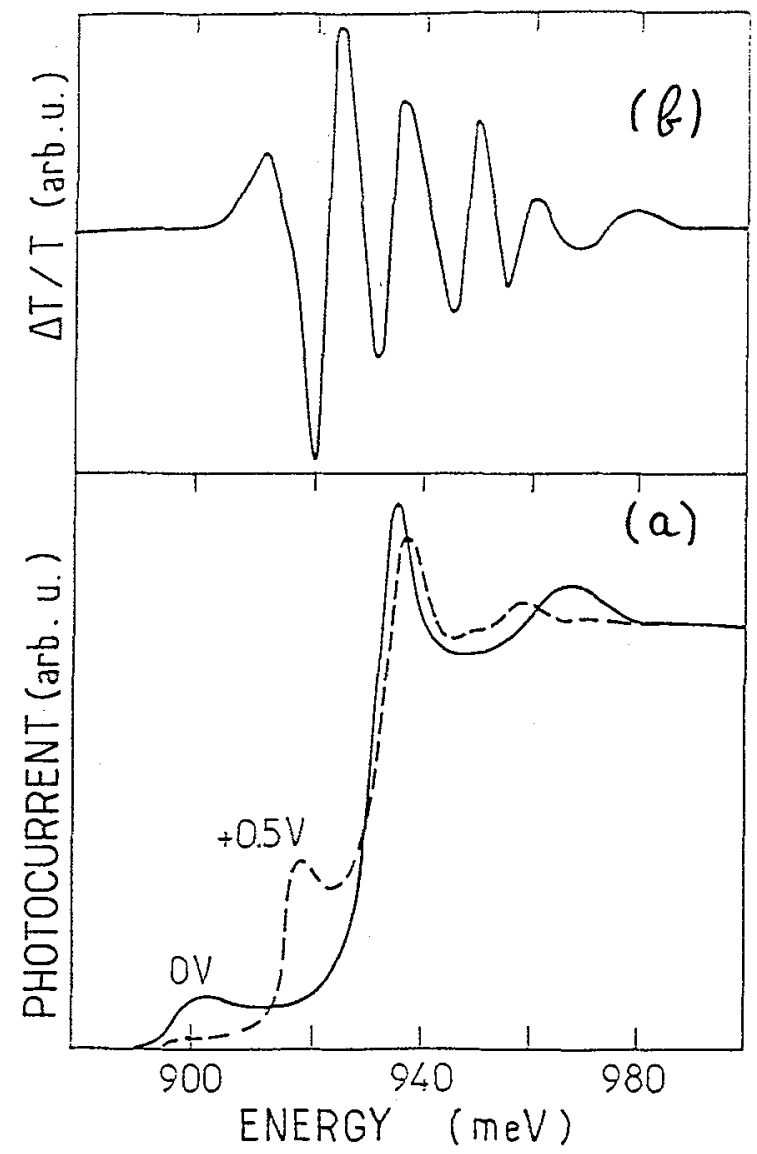

Fig. 1 (a) Low temperature photocurrent spectra at $0 \mathrm{~V}$ (solid line) and $0.5 \mathrm{~V}$ forward bias (dashed line) and (b) differential transmission spectrum. The transmission of the low power working light is modulated by a $0.5 \mathrm{~mW}$ He-Ne laser chopped at $75 \mathrm{~Hz}$.

In the context of optical switching, the blueshift modulation is essential because it provides a positive feedback mechanism which can lead to bistability. Indeed, if we increase the intensity of the weakly absorbed incident beam at $920 \mathrm{meV}$, more and more photocarriers will be created, which will screen the electric field and increase the absorption: for a sufficient intensity, absorption will cause its own increase, and the system may switch from the weakly absorbing "high field" regime to the highly absorbing near fiatband regime. This actually occurs in our sample, as illustrated in Fig. 2 which shows the transmitted intensity versus the incident intensity at various photon energies: outside the region of blueshift modulation, we get nearly straigth lines, with a slope 1 in the transparency region and a smaller slope in the absorbing region ${ }^{10}$. On the contrary, near $920 \mathrm{meV}$, the curves show a clear non-linear behavior and a nice hysteresis loop is observed at $h v=920 \mathrm{meV}$. To the best of our knowledge, the input power at the middle of the loop, $1 \mu \mathrm{W} / \mathrm{mm}^{2}$, is smaller than any bistability threshold ever reported. 


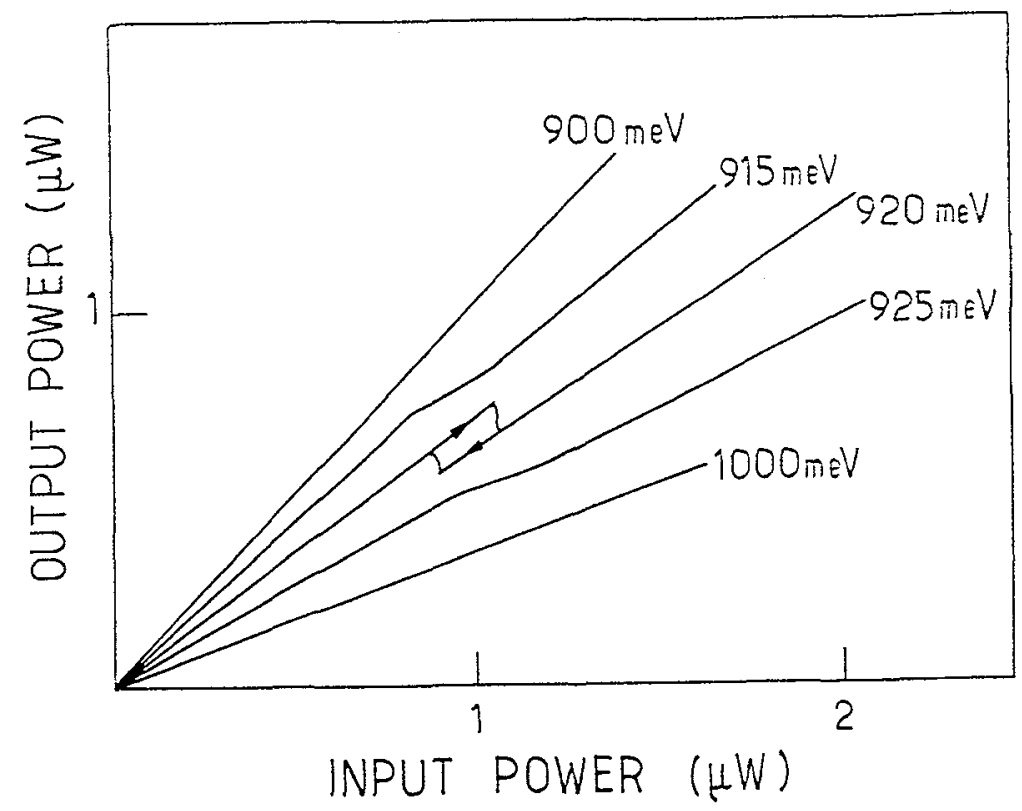

Fig. 2 Transmitted versus incident intensities at various photon energies. The vertical axis has been scaled to ensure a slope equal to unity in the transparency region.

The way this device works has strong analogies with the SEED, but also the major difference that no load circuit is needed. The voltage across the superlattice can be phenomenologically described by:

$$
V=V_{0}\left\{1-\exp \left(-\gamma \alpha I_{\text {in }}\right)\right\}
$$

where $\mathrm{V}_{0} \approx 0.9 \mathrm{~V}$ is the built-in voltage, $\alpha$ the absorption, $\gamma$ a numerical constant and $\mathrm{I}_{\text {in }}$ the incident power. This gives the equivalent of a load line in the form:

$$
\alpha=\ln \left(\mathrm{V}_{0} /\left(\mathrm{V}_{0}-\mathrm{V}\right)\right\} /\left(\gamma \mathrm{I}_{\mathrm{in}}\right)
$$

In Fig. 3, we have plotted the $\alpha(V)$ curves measured directly from the combination of transmission and photocurrent data, for various photon energies, together with the load lines Eq. 2, obtained for increasing values of $\gamma \mathrm{I}_{\mathrm{in}}$. It clearly appears that in the transparency region ( $\mathrm{hv}=905 \mathrm{meV}$ ) or far above the bandgap ( $h v=990 \mathrm{meV}$ ) there exists only one intersection describing the only stable state of the system. On the contrary, for $h v=921 \mathrm{meV}$, there is a domain of $\gamma \mathrm{I}_{\mathrm{in}}$ where three intersections exist. An argument quite similar to the original discussion of Miller et al. ${ }^{3}$ proves that the central point is unstable, while the two others correspond to the stable states in the hysteresis loop. Hence, our device is basically a "wireless superlattice SEED".

While our observation of extremely low power all-optical bistability certainly opens new perspectives, we are still far from an operational device for which a number of technical requirements exist. In particular, room temperature operation and larger contrast in the bistability loop are obviously necessary. As for the first point, we have observed that the differential transmission signal saturates at a similar value at low and room temperatures, which means that the modulation depth is similar. The main difference is that a much higher power $(\approx 100 \mathrm{~mW})$ is needed at room temperature. This is certainly because the faster diffusion of carriers at room temperature and/or the activation of alternative vertical transport mechanisms (possibly associated with extended defects in our "jumbo" diode) decrease the efficiency of screening by photocarriers. 


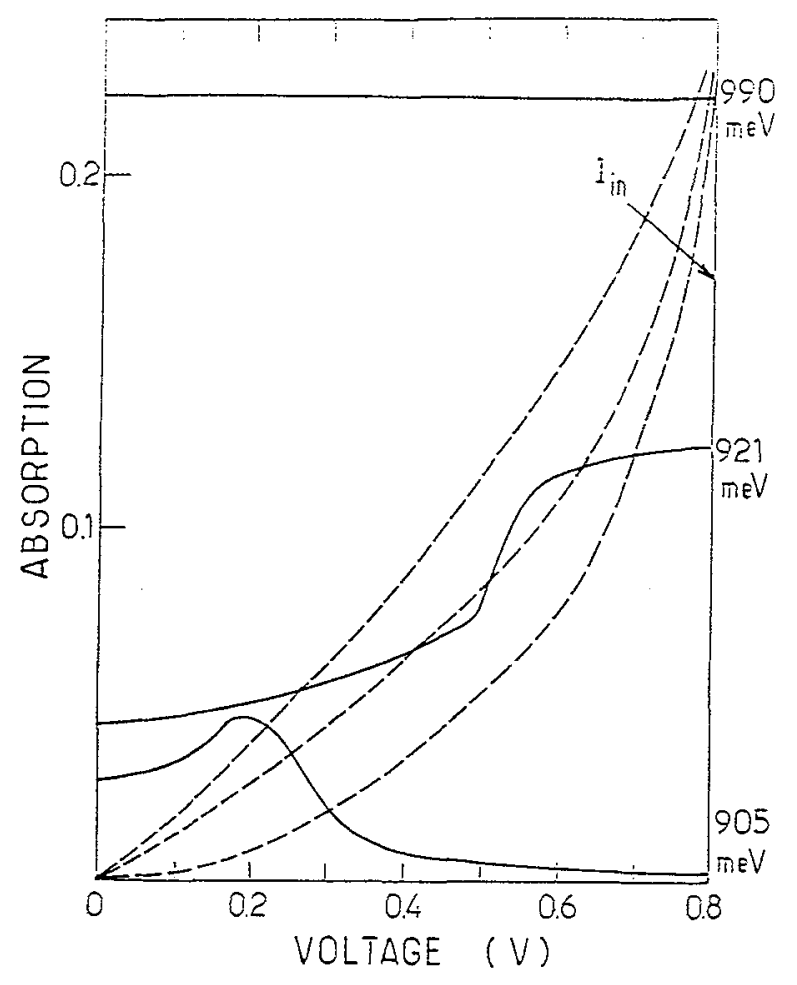

Fig. 3 Absorption versus voltage curves (solid lines) for various photon energies and "load lines" deduced from Eq. 1 (dashed lines) for increasing values of the incident power.

This merely amounts to a much smaller factor $\gamma$ (Eq.1) at $300 \mathrm{~K}$ than at $2 \mathrm{~K}$. In this case, our bistable device should also work at room temperature, at the expense of larger input power. Let us stress that the fabrication of more realistic pixels (eg $30 \times 30 \mu \mathrm{m}^{2}$ ) will keep the input power in sub-microwatt range, even if $\gamma$ is reduced by a factor 100 . Note that the use of asymmetric Fabry-Pérot cavities may solve the modulation contrast even more easily than it did for conventional SEEDs ${ }^{11}$.

In conclusion, we have observed very low power all-optical bistability in a wireless superlattice SEED operating at $1.5 \mu \mathrm{m}$. As the Wannier-Stark effect is a genuine band effect, these observations should be easily extended to other systems, including GaAs-AlGaAs or large gap II-VI heterostructures. In principle, it should also be possible to design isolated quantum well structures showing the wireless SEED behavior, but the Wannier-Stark effect has a-priori avantages over the Quantum Confined Stark Effect in terms of low field and high modulation contrast.

Acknowledgements: we have benefitted from discussions with Dr. E. Bigan. LPMC-ENS and CNETBagneux are Unités Associées at CNRS. This work is supported in part by DRED.

\section{References:}

1 H.M. Gibbs, Optical bistability: controlling light with light (Academic Press, New York, 1985)

2 J.L. Oudar, R. Kuszelewicz, B. Sfez, J.C. Michel and R. Planel, Optical and Quantum Electronics 24, S 193 (1992)

3 D.A.B. Miller, D.S. Chemla, T.C. Damen, T.H. Wood, C.A. Burrus, A.C. Gossard and W. Wiegmann, IEEE Journal of Quantum Electronics QE 21,1462 (1985)

4 A. Lentine, H.S. Hinton, D.A.B. Miller, J.E. Henry, J.E. Cunningham and L.M.F. Chirovski, IEEE Journal of Quantum Electronics QE 25,1928 (1989)

$5 \quad$ F.B. McCormick et al., Optical Engineering 31, 2697 (1992)

6 E. Bigan, M. Allovon, M. Carré and P. Voisin, Appl. Phys. Lett. 57, 327 (1990)

7 J. Bleuse, G. Bastard and P. Voisin, Phys. Rev. Lett. 60, 220 (1988)

8 E.E. Mendez, F. Agullo-Rueda, and J.M. Hong, Phys. Rev. Lett. 60, 2426 (1988)

9 J. Bleuse, P. Voisin, M. Allovon and M. Quillec, Appl. Phys. Lett. 53, 2632 (1988)

10 The slope at $h v=1 \mathrm{eV}$ should be 0.8 , but there is an additional absorption in the $1000 \AA$ thick InGaAs contact layer, and the transmission is actually smaller.

11 K.K. Law, R.H. Yan, L.A. Coldren and J.L. Merz, Appl. Phys. Lett. 571345 (1990) 